\title{
Thoracotomy in refractory gestational trophoblastic neoplasia with lung metastasis after normalization of serum beta human chorionic gonadotropin ( $\beta-\mathrm{hCG}$ ) with salvage chemotherapy
}

This article was published in the following Dove Press journal:

OncoTargets and Therapy

29 January 2014

Number of times this article has been viewed

\section{Fengzhi Feng \\ Huiying $\mathrm{Hu}$ \\ Lei Wu \\ Tong Ren \\ Xirun Wan \\ Yang Xiang}

Department of Obstetrics and Gynecology, Peking Union Medical College Hospital, Chinese Academy of Medical Sciences, Beijing, People's Republic of China
Correspondence: Fengzhi Feng; Yang Xiang

Department of Obstetrics and Gynecology, Peking Union Medical College Hospital, Chinese Academy of Medical Sciences, No. I Shuai Fu Yuan, Wang Fu Jing Street, Beijing 100730, People's Republic of China

Tel +861069156218

Fax +86 10 6512 4875

Email fengfz1969@sina.com (FZ Feng); xiangyang65@gmail.com (Y Xiang)
Objective: To assess the need for pulmonary surgery in the treatment of refractory gestational trophoblastic neoplasia with lung metastasis after normalization of serum beta human chorionic gonadotropin ( $\beta$-hCG) level with salvage chemotherapy.

Materials and methods: A review of medical records of patients with refractory gestational trophoblastic neoplasia who underwent pulmonary surgery and received combined chemotherapy between January 1995 and December 2008 at the Peking Union Medical College Hospital was retrospectively performed. The positive pathologic findings in surgical specimens were defined as trophoblastic cells documented in the specimen. Pathologic findings were reported.

Results: There were 21 patients with preoperative normal $\beta$-hCG. Of 21 patients, six $(28.6 \%)$ had positive pathologic findings. The positive pathologic findings remained at $27.3 \%$ in 11 patients who had received no less than two cycles of consolidation chemotherapy before pulmonary surgery. Univariate analysis found that no variables in patient characteristics were associated with pathologic findings. At the median follow-up of 78 months (9-186 months), $85.7 \%$ (18 of 21) patients were alive, and no statistical difference was observed in the disease-free survival between the patients with positive and negative pathologic findings. The 5-year overall survival was $72.2 \%$.

Conclusion: Pulmonary surgery is valuable in the treatment of refractory patients with lung metastasis after normalization of serum $\beta$-hCG level following salvage chemotherapy, irrespective of viable trophoblasts in surgical specimens. Further study will be necessary to clarify the importance of this observation.

Keywords: gestational trophoblastic neoplasia, refractory, pulmonary surgery

\section{Introduction}

Gestational trophoblastic neoplasia (GTN) is prone to hematogenous metastasis, most commonly to the lungs. Pulmonary metastases occur in as many as $70 \%$ of patients with GTN, and $90 \%$ of these patients are cured with chemotherapy. ${ }^{1-3}$ However, a small minority of patients have refractory GTN associated with lung metastasis after a failure of first line chemotherapy. At this time, lobectomy or partial thoracotomy has been supported in several small sample studies and case reports, particularly in patients with abnormal serum beta human chorionic gonadotropin ( $\beta$-hCG) levels. ${ }^{2,4-8}$ In addition, in our previous study, although we found further treatment was not needed for patients 
with complete response to the first line chemotherapy and residual pulmonary lesion, trophoblastic tumor cells existed in $35.7 \%$ (10/28) refractory patients who underwent pulmonary surgery. ${ }^{2}$ Salvage chemotherapy, often combined with surgical resection of sites of persistent lesion, will result in cure for the majority of patients with refractory GTN. For patients with normalization of serum $\beta$-hCG after salvage chemotherapy, the impact of surgery on patient outcome was usually defined as not assessable. ${ }^{9}$ To date, there is limited data assessing the need for pulmonary surgery in refractory patients with residual pulmonary lesion after normalization of serum $\beta$-hCG with salvage chemotherapy. Despite normalization of serum $\beta-\mathrm{hCG}$ with salvage chemotherapy in refractory patients, normal $\beta$-hCG levels cannot guarantee absence of active trophoblastic cells, which could be the origin of relapse. Thus, is it necessary to perform surgery on the refractory patient with normal $\beta$-hCG level after salvage chemotherapy? The answer remains uncertain. This prompted us to conduct this retrospective study of patients with refractory GTN associated with pulmonary lesion. The goal of the present study is to determine whether pulmonary surgery is necessary when the serum $\beta$-hCG level is normalized after salvage chemotherapy.

\section{Materials and methods}

This study was retrospective and conducted between January 1995 and December 2008 at the Peking Union Medical College Hospital (PUMCH). The cutoff date of follow-up was December 2012.

Patients with GTN were identified through review of institutional databases. Eligibility criteria included the following: patients with refractory GTN during first line chemotherapy who underwent lobectomy or partial thoracotomy at the PUMCH, and where postoperative chemotherapy and follow-up were performed at the PUMCH. Refractory GTN was defined according to following criteria: a plateau $(<1$ logarithmic fall in the $\beta$-hCG level) or an increased $\beta$-hCG level despite receiving at least two consecutive cycles of standard chemotherapy. The patients with placental site trophoblastic tumors or epithelioid trophoblastic tumors were excluded from this analysis because they had rather variable chemosensitivity. All patients provided written informed consent before starting the treatment at the PUMCH.

All patients with refractory GTN received salvage chemotherapy with multidrug regimens. Several multidrug chemotherapy regimens were used, based on the previous chemotherapy protocols that patients had received. Salvage chemotherapy regimens commonly included: vincristine, floxuridine, dactinomycin, and etoposide; etoposide, methotrexate, and dactinomycin, alternating with cyclophosphamide and vincristine; or etoposide, methotrexate, and dactinomycin, alternating with etoposide and cisplatin. Serum $\beta$-hCG levels were measured before each cycle of chemotherapy. Chemotherapy was changed if serum $\beta$-hCG levels reached a plateau or began to increase for two consecutive cycles of chemotherapy. The patients received three or four cycles of consolidation chemotherapy after normalization of serum $\beta$-hCG.

For each patient, all available preoperative data were collected, including age, type of antecedent pregnancy, International Federation of Gynecology and Obstetrics stage and score, the number of preoperative chemotherapy cycles, preoperative disease sites, and preoperative $\beta$-hCG level. All lobectomies or partial thoracotomies were performed at the PUMCH. Before deciding to perform surgical resection, computed tomography or magnetic resonance imaging of the brain, chest, and abdomen, and ultrasonography of the pelvis were performed to document the site and number of lung metastases and the disease outside the lung. To minimize the possibility of inducing metastasis of trophoblastic cells by surgical manipulation of tissue, surgical procedures were usually performed during chemotherapy.

All surgical specimens were analyzed. Positive pathologic findings of the resected specimen were defined as trophoblastic cells documented in the specimen, whereas necrotic or hemorrhagic tissue, fibrosis nodules, or no trophoblastic cells was defined as negative pathologic findings. Serum complete remission was diagnosed after weekly $\beta$-hCG level was within the normal range ( $\leq 2 \mathrm{mIU} / \mathrm{mL}$ ) for three consecutive weeks during treatment. After the completion of treatment, serum $\beta$-hCG level was measured weekly for 4 weeks, then biweekly for up to 3 months, followed by monthly measurements for the rest of the first year, then every 3 months in the second year, and every 6 months to 1 year thereafter.

Demographic and baseline characteristics were summarized by using medians (with ranges) for continuous variables and proportions for categorical variables. The correlation between pathologic findings and clinical factors was done using the chi-square test. Survival analysis was performed using Kaplan-Meier methods. Differences were considered statistically significant at $P<0.05$. All analyses were carried out using the Statistical Package for Social Sciences (version 12.0.1; SPSS Inc., Chicago, IL, USA).

\section{Results}

\section{Patient characteristics}

Twenty-one patients with refractory GTN who underwent lobectomy had preoperative normal serum $\beta$-hCG 
levels between January 1995 and December 2008. Patient characteristics are summarized in Table 1. At the time of pulmonary lobectomy, all patients were heavily pretreated with chemotherapy. The median number of preoperative regimens was four and ranged from two to six, and $85.7 \%$ of patients received at least three chemotherapy regimens. Nineteen $(90.5 \%)$ of the patients had a high-risk GTN.

\section{Pathology}

Of the 21 patients who underwent a resection of pulmonary metastases, six (28.6\%) had positive pathologic findings. Clinical characteristics and pathologic findings in the patients are summarized in Table 2. No variables in patient characteristics were associated with pathologic findings. Moreover, three $(27.3 \%)$ of 11 patients who had no less than two cycles

Table I Patient characteristics

\begin{tabular}{|c|c|}
\hline Variable & $\mathbf{N}(\%)$ \\
\hline Median age (range) (years) & $29(23-40)$ \\
\hline \multicolumn{2}{|l|}{ Antecedent pregnancy } \\
\hline Mole & $6(28.6 \%)$ \\
\hline Abortion & $6(28.6 \%)$ \\
\hline Term & $9(42.8 \%)$ \\
\hline \multicolumn{2}{|l|}{ Preoperative chemotherapy } \\
\hline Median cycles (range) & $13(4-29)$ \\
\hline Median regimens (range) & $4(2-6)$ \\
\hline \multicolumn{2}{|l|}{ Number of preoperative chemotherapy regimens } \\
\hline 2 & $3(14.3 \%)$ \\
\hline 3 & $4(19.0 \%)$ \\
\hline 4 & 7 (33.3\%) \\
\hline 5 & $6(28.6 \%)$ \\
\hline 6 & I (4.8\%) \\
\hline \multicolumn{2}{|c|}{ Type of preoperative chemotherapy regimens $(\mathrm{N}=72)$} \\
\hline $5-\mathrm{FU}$ & 7 \\
\hline Methotrexate & 2 \\
\hline Dactinomycin & 1 \\
\hline FAV & 20 \\
\hline FAEV & 21 \\
\hline EMA-CO & 10 \\
\hline EMA-EP & 3 \\
\hline Methotrexate + etoposide + dactinomycin & 2 \\
\hline Cisplatin + etoposide + dactinomycin & 1 \\
\hline Cisplatin + etoposide + bleomycin & 2 \\
\hline Cisplatin + paclitaxel & 3 \\
\hline \multicolumn{2}{|l|}{ Staging } \\
\hline III & 17 (8I\%) \\
\hline IV (brain metastasis) & $4(19 \%)$ \\
\hline \multicolumn{2}{|l|}{ Prognostic score (FIGO) } \\
\hline$\leq 6$ & $2(9.5 \%)$ \\
\hline$>6$ & $19(90.5 \%)$ \\
\hline
\end{tabular}

Abbreviations: 5-FU, 5-fluorouracil; EMA-CO, etoposide, methotrexate, and dactinomycin, alternating with cyclophosphamide and vincristine; EMA-EP, etoposide, methotrexate, and dactinomycin, alternating with etoposide and cisplatin; FAV, 5-FU or floxuridine + dactinomycin + vincristine; FAEV, 5-FU or floxuridine + dactinomycin + etoposide + vincristine; FIGO, International Federation of Gynecology and Obstetrics.
Table 2 The clinical characters and pathologic results of patients with normal preoperative beta human chorionic gonadotropin $(\mathrm{N}=2 \mathrm{l})$

\begin{tabular}{|c|c|c|c|}
\hline \multirow[t]{2}{*}{ Variable } & \multicolumn{2}{|c|}{ Pathological results } & \multirow[t]{2}{*}{$P$-value } \\
\hline & $\begin{array}{l}\text { Positive } \\
(\mathrm{N}=6)\end{array}$ & $\begin{array}{l}\text { Negative } \\
(\mathrm{N}=15)\end{array}$ & \\
\hline Age, years (median) & $31(3.8)$ & $28(7.4)$ & 0.334 \\
\hline $\begin{array}{l}\text { Prognostic score } \\
\text { (FIGO) (median) }\end{array}$ & $9(2.3)$ & $9(2.6)$ & 0.439 \\
\hline$\leq 6$ & 0 & 2 & \\
\hline$>6$ & 6 & 13 & \\
\hline $\begin{array}{l}\text { Preoperative courses of } \\
\text { chemotherapy (median) }\end{array}$ & $13(6.5)$ & $12(5.2)$ & 0.456 \\
\hline \multicolumn{4}{|l|}{ Antecedent pregnancy } \\
\hline Mole & I & 5 & 0.623 \\
\hline Non-mole & $5(37.5 \%)$ & $10(62.5 \%)$ & \\
\hline \multicolumn{4}{|l|}{ Staging } \\
\hline III & $4(23.5 \%)$ & $13(76.5 \%)$ & 0.316 \\
\hline IV & 2 & 2 & \\
\hline \multicolumn{4}{|c|}{ Site of pulmonary metastasis } \\
\hline Bilateral & I & I & 0.500 \\
\hline Unilateral & $5(26.3 \%)$ & 14 (73.7\%) & \\
\hline \multicolumn{4}{|c|}{ Preoperative consolidation chemotherapy courses } \\
\hline$<2$ cycles & $3(30 \%)$ & 7 (70\%) & 0.633 \\
\hline$\geq 2$ cycles & $3(27.3 \%)$ & $8(73.7 \%)$ & \\
\hline
\end{tabular}

Abbreviation: FIGO, International Federation of Gynecology and Obstetrics.

of consolidation chemotherapy before surgery still had positive pathologic findings.

\section{Survival}

Postoperatively, one of the 21 patients who underwent pulmonary surgery did not receive further treatment and died. This patient died due to cerebral hemorrhage at 11 days after surgery.

At the time of analysis, at the median follow-up of 78 months (9-186 months), of the 20 patients who completed treatment, three had relapsed disease, including two with negative pathologic findings and one with positive pathologic findings. Only one patient achieved a durable remission by further salvage chemotherapy combined with hysterectomy, whereas two ultimately died of GTN. Overall, at the time of analysis, 18 patients (85.7\%) were alive without GTN (Figure 1). The survival rate of patients with positive and negative pathologic findings was $83.3 \%$ (five of six patients) and $86.7 \%$ (13 of 15 patients), respectively ( $P=0.658)$. The 5-year overall survival was $72.2 \%$ (Figure 2 ).

\section{Discussion}

Treatment of GTN represents one of the most dramatic successes of chemotherapy in the treatment of human malignancy. However, the primary refractory rate to initial chemotherapy 


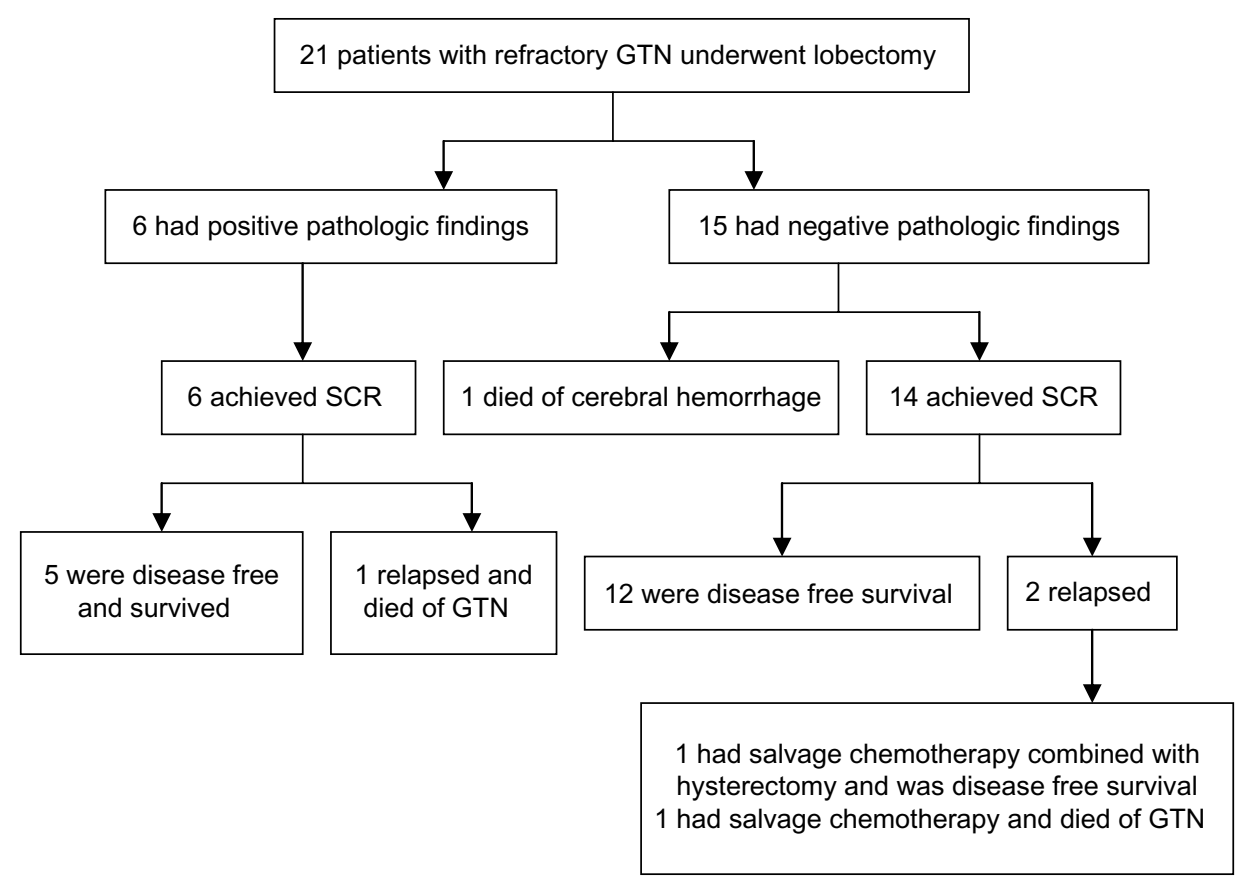

Figure I Patient treatment profile.

Abbreviations: GTN, gestational trophoblastic neoplasia; SCR, serum complete remission.

of patients with nonmetastatic low risk, metastatic low risk, and high risk GTN is $10 \%-20 \%, 30 \%-50 \%$, and $20 \%-30 \%$, respectively. ${ }^{10}$ Salvage chemotherapy, often combined with surgical resection of sites of persistent lesion, will result in cure for a majority of patients with refractory GTN. The impact of surgery on the patients' outcome was determined using the effect of the operation on the $\beta$-hCG levels. The impact of surgery was usually defined as effective when the $\beta$-hCG clearly decreased and achieved normal values in the postoperative periods, or not assessable when the $\beta$-hCG was within the

\section{Survival function}

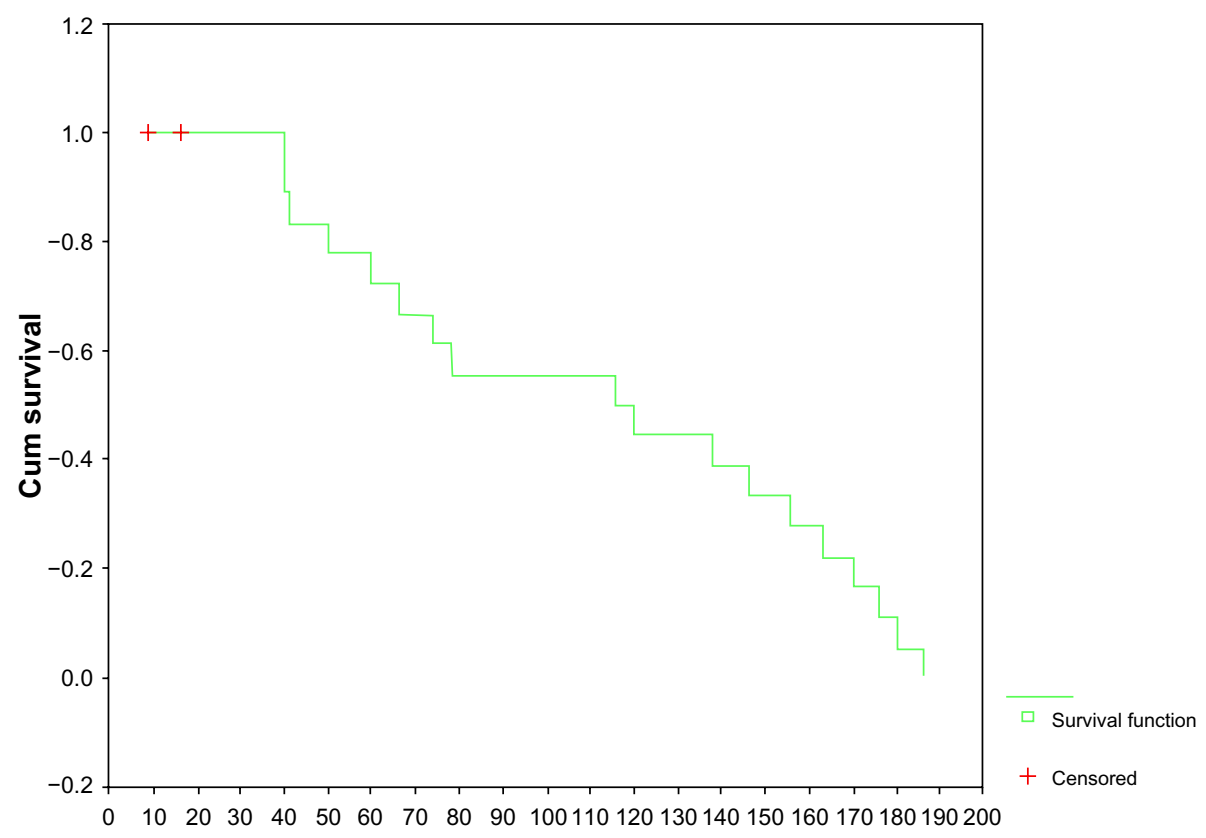

Time after diagnosis (months)

Figure 2 Kaplan-Meier estimate of overall survival. 
normal range at the time of surgery. ${ }^{9}$ However, publications examining the impact of surgery often do not distinguish between the patients with and without normalization of serum $\beta$-hCG. ${ }^{2,-8}$ Therefore, little is known about the role of salvage surgery in patients with normalization of serum $\beta$-hCG following salvage chemotherapy.

Refractory GTN associated with lung metastasis has been attributed to an ineffective concentration of drugs reaching the lung lesion because of central necrosis, or encasement of tumor by fibrin, or the presence of a clone(s) of resistant cells that pre-existed within the lung lesion or that developed following chemotherapy. ${ }^{10,11}$ Several retrospective studies have demonstrated that pulmonary surgery is effective in selected patients with refractory GTN associated with lung metastasis, despite the series often being small in size..$^{2,4-8}$ Fleming et al reviewed their experience with pulmonary surgery in 11 patients with refractory GTN and proposed the following criteria for successful resection: good surgical candidate, stage III disease, a preoperative hCG of less than $1500 \mathrm{mIU} / \mathrm{mL}$, and a solitary lung nodule resistant to chemotherapy. ${ }^{8}$ Similarly, Lurain et al reported that prompt regression of $\beta$-hCG within 1-2 weeks of surgical resection predicted a favorable outcome. ${ }^{6}$ In our previous study, we found that $91.5 \%$ of patients with refractory GTN who underwent surgical management combined with chemotherapy achieved remission when they had a preoperative serum $\beta$-hCG level of less than $10 \mathrm{IU} / \mathrm{L}$ (normal serum $\beta$-hCG level $\leq 2 \mathrm{mIU} / \mathrm{mL}) .{ }^{5}$ However, none of these studies provides any additional data to that presented here concerning the impact of surgery on patients with normal serum $\beta$-hCG level following chemotherapy.

In our previous study, although we found that further treatment is not needed for patients with normal $\beta$-hCG level and residual lung tumor, it refers to nonrefractory or invasive mole patients. In five chemoresistant and eight nondrug-resistant patients who received pulmonary surgery, positive pathologic findings were found in $60 \%$ (three of five) and $12.5 \%$ (one of eight) of patients, respectively. ${ }^{12}$ For chemoresistant and recurrent patients, it is difficult to thoroughly eliminate the tumor cells by chemotherapy alone. Accordingly, we believe pulmonary surgery is necessary for refractory patients to avoid progression of disease. In the present study, six of 21 patients with normal preoperative $\beta$-hCG level had positive pathologic findings. Moreover, of 11 patients who actually had no less than two cycles of consolidation chemotherapy before lobectomy, 27.3\% (three of eleven) still had positive pathologic findings. This finding suggests that some patients with apparently refractory GTN do benefit from salvage surgery even if normalization of serum $\beta-\mathrm{hCG}$ is reached by chemotherapy. Thus, salvage surgery is indicated for refractory patients with normal preoperative $\beta$-hCG level after salvage chemotherapy.

Doumplis et al described that adjuvant chemotherapy was not administrated when no viable trophoblast was identified in surgical specimens. ${ }^{13}$ However, in this study, two patients with negative pathologic findings who received postoperatively chemotherapy still had relapse. In addition, the survival rate of refractory patients was not statistically significantly different between patients with positive and negative pathologic findings. Therefore, we suggest that postoperative chemotherapy should be continued until the chemotherapy scheme is completed, irrespective of viable trophoblast in surgical specimens.

Surgery clearly played a significant role in achieving complete remission in selected patients. Patients with only isolated and unilateral metastatic disease in the lung were more likely to benefit from surgical resection with either lobectomy or partial thoracotomy when compared with those with bilateral or multiple lung lesions or those who had more than one disease site. ${ }^{5,8}$ However, in the present study, four patients with brain and lung metastases who underwent thoracotomy for possible chemoresistant lung disease achieved serum complete remission. It should be noted that although refractory patients would have more than one disease site on metastatic evaluation, thoracotomy is indicated for patients with lung lesion. In addition, as a specialist center for GTN, a group from the Charing Cross Hospital, UK, have reported on the outcome of patients with chemorefractory GTN who received initial treatment at the Charing Cross Hospital. The overall 5-year survival for patients with chemorefractory GTN was $43 \% .{ }^{4}$ In this study, the outcome of patients with refractory GTN who underwent pulmonary surgery had good prognosis as the overall 5-year survival was $72.2 \%$. Thus, the importance of salvage surgery for patients with refractory GTN was underlined.

In conclusion, we found $27.3 \%$ of positive pathologic findings in refractory patients with lung metastasis who underwent pulmonary surgery after normalization of serum $\beta$-hCG level for no less than two cycles of consolidation chemotherapy. This result emphasizes the importance of combined salvage surgery, rather than salvage chemotherapy alone in refractory patients in whom normalization of serum $\beta$-hCG level is attained after salvage chemotherapy, to reduce the possibility of recurrence. We propose that salvage surgery is indicated for refractory patients with normal preoperative $\beta$-hCG level and localized pulmonary lesion, although 
further study will be necessary to clarify the importance of this observation.

\section{Funding}

The study was financially supported by the Key Projects in National Science and Technology Pillar Program in the 11th 5-year plan period (grant No 2008BAI57B05).

\section{Acknowledgment}

We thank all of the patients. Without their continuing data collection, this study would not have been possible.

\section{Disclosure}

The authors declare no conflict of interest in this work.

\section{References}

1. El-Helw LM, Hancock BW. Treatment of metastatic gestational trophoblastic neoplasia. Lancet Oncol. 2007;8(8):715-724.

2. Cao Y, Xiang Y, Feng F, Wan X, Yang X. Surgical resection in the management of pulmonary metastatic disease of gestational trophoblastic neoplasia. Int J Gynecol Cancer. 2009;19(4):798-801.

3. Hoekstra AV, Lurain JR, Rademaker AW, Schink JC. Gestational trophoblastic neoplasia: treatment outcomes. Obstet Gynecol. 2008; 112(2 Pt 1):251-258.

4. Powles T, Savage PM, Stebbing J, et al. A comparison of patients with relapsed and chemo-refractory gestational trophoblastic neoplasia. $\mathrm{Br} \mathrm{J}$ Cancer. 2007;96(5):732-737.
5. Feng F, Xiang Y, Li L, Wan X, Yang X. Clinical parameters predicting therapeutic response to surgical management in patients with chemotherapy-resistant gestational trophoblastic neoplasia. Gynecol Oncol. 2009;113(3):312-315.

6. Lurain JR, Singh DK, Schink JC. Role of surgery in the management of high-risk gestational trophoblastic neoplasia. J Reprod Med. 2006;51(10):773-776.

7. Lehaman E, Gershenson DM, Burke TW, Levenback C, Silva EG, Morris M. Salvage surgery for chemorefractory gestational trophoblastic disease. J Clin Oncol. 1994;12(12):2737-2742.

8. Fleming EL, Garrett L, Growdon WB, et al. The changing role of thoracotomy in gestational trophoblatic neoplasia at the New England Trophoblastic Disease Center. J Report Med. 2008;53(7): 493-498.

9. Newlands ES, Mulholland PJ, Holden L, Seckl MJ, Rustin GJ. Etoposide and cisplatin/etoposide, methotrexate, and actinomycin D (EMA) chemotherapy for patients with high-risk gestational trophoblastic tumors refractory to EMA/cyclophosphamide and vincristine chemotherapy and patients presenting with metastatic placental site trophoblastic tumors. J Clin Oncol. 2000;18(4):854-859.

10. Feng F, Xiang Y. Surgical management of chemotherapy-resistant gestational trophoblastic neoplasms. Expert Rev Anticancer Ther. 2010;10(1):71-80.

11. Hanna RK, Soper JT. The role of surgery and radiation therapy in the management of gestational trophoblastic disease. Oncologist. 2010;15(6):593-600.

12. Yang J, Xiang Y, Wan X, Yang X. The prognosis of gestational trophoblastic neoplasia patient with residual lung tumor after completing treatment. Gynecol Oncol. 2006;103(2):479-482.

13. Doumplis D, Al-khatib K, Sieunarine K, et al. A review of the management by hysterectomy of 25 cases of gestational trophoblastic tumours from March 1993 to January 2006. BJOG. 2007;114(9): $1168-1171$.
OncoTargets and Therapy

\section{Publish your work in this journal}

OncoTargets and Therapy is an international, peer-reviewed, open access journal focusing on the pathological basis of all cancers, potential targets for therapy and treatment protocols employed to improve the management of cancer patients. The journal also focuses on the impact of management programs and new therapeutic agents and protocols on

\section{Dovepress}

patient perspectives such as quality of life, adherence and satisfaction The manuscript management system is completely online and includes a very quick and fair peer-review system, which is all easy to use. Visit http://www.dovepress.com/testimonials.php to read real quotes from published authors. 